\title{
DETECTION OF VERY RARE ISOTOPES BY LASER COLLINEAR RESONANT IONIZATION OF FAST ATOMS
}

\author{
Yu.A. KUDRYAVTSEV \\ Institute of Spectroscopy, USSR Academy of Sciences, 142092 Troitsk, Moscow Region, \\ Russia
}

\begin{abstract}
Results are presented of the work on the development of a method for detecting ultra rare isotopes, based on the collinear isotope-selective laser ionization of atoms in a fast beam. The rare isotope ${ }^{3} \mathrm{He}$ was detected at a selectivity of $10^{9}$ and isotope-selective ionization of krypton atoms was demonstrated. A scheme is suggested for detecting the rare radioactive isotopes ${ }^{85} \mathrm{Kr}$ and ${ }^{81} \mathrm{Kr}$. The technique has been exploited for the measurement of hyperfine structures and isotope shifts of unstable $\mathrm{Yb}$ isotopes.
\end{abstract}

\section{Introduction}

In the world around us, there are isotopes whose concentration amounts to a neglible fraction $\left(<10^{-10}\right)$ of that of the stable isotopes of the same elements.

There are a fairly large number of rare isotopes of cosmic origin, particularly those formed in the upper atmosphere as a result of nuclear reactions caused by cosmic rays. They include such isotopes as ${ }^{10} \mathrm{Be},{ }^{14} \mathrm{C},{ }^{26} \mathrm{Al}$. These isotopes form in the upper atmosphere, precipitate, and accumulate on the Earth's surface and ocean bottom.

There are also a number of radioactive isotopes of technological origin (e.g. from nuclear tests and the nuclear power industry). The most abundant and dangerous artificial radioisotopes are ${ }^{90} \mathrm{Sr}$ and ${ }^{137} \mathrm{Cs}$. These isotopes can accumulate in the living organism, ${ }^{90} \mathrm{Sr}$ concentrating in the bones, where it replaces calcium and stable strontium, and ${ }^{137} \mathrm{Cs}$ in muscles, replacing potassium and stable cesium.

Many problems in nuclear physics also involve the determination of ultra-low concentrations of isotopes. For example, such a problem arises in the detection of solar neutrinos (the isotopes involved being ${ }^{205} \mathrm{~Pb},{ }^{81} \mathrm{Kr},{ }^{98} \mathrm{Tc}$ and ${ }^{71} \mathrm{Ga}$ ), in the search for exotic fractionally charged particles and in the measurement of radionuclide production cross-sections and long lifetimes.

High selectivity and sensitivity are also required for obtaining information on ground-state properties of exotic nuclei produced at on-line mass-separator facilities.

The development of laser techniques for detecting rare long-lived isotopes has recently reccived growing interest $[1,2]$. If the isotope shifts in the absorption spectra of elements are very small, the required ultra-high isotopic selectivity can 
be ensured either by multiple-step laser excitation or by repeated interaction of the laser radiation with the atoms of interest.

The following methods have been suggested or already experimentally investigated for the detection of rare isotopes: laser resonance atomic photoionization [3-5], photon burst [6-8], selective atomic deceleration [9], selective atomic deflection $[10,11]$, ground state depletion $[12,13]$, and collinear laser photoionization of atoms in an accelerated beam $[14-16]$. Most of these spectroscopic methods have been developed for investigation of nuclear ground-state properties of isotopes far off stability. Review of these methods can be found in $[17,18]$.

This paper describes a rather universal method of detecting rare isotopes, based on collinear isotope-selective laser ionization of fast atoms in a beam. The idea of the method is as follows [14]. The atoms of all isotopes in the substance

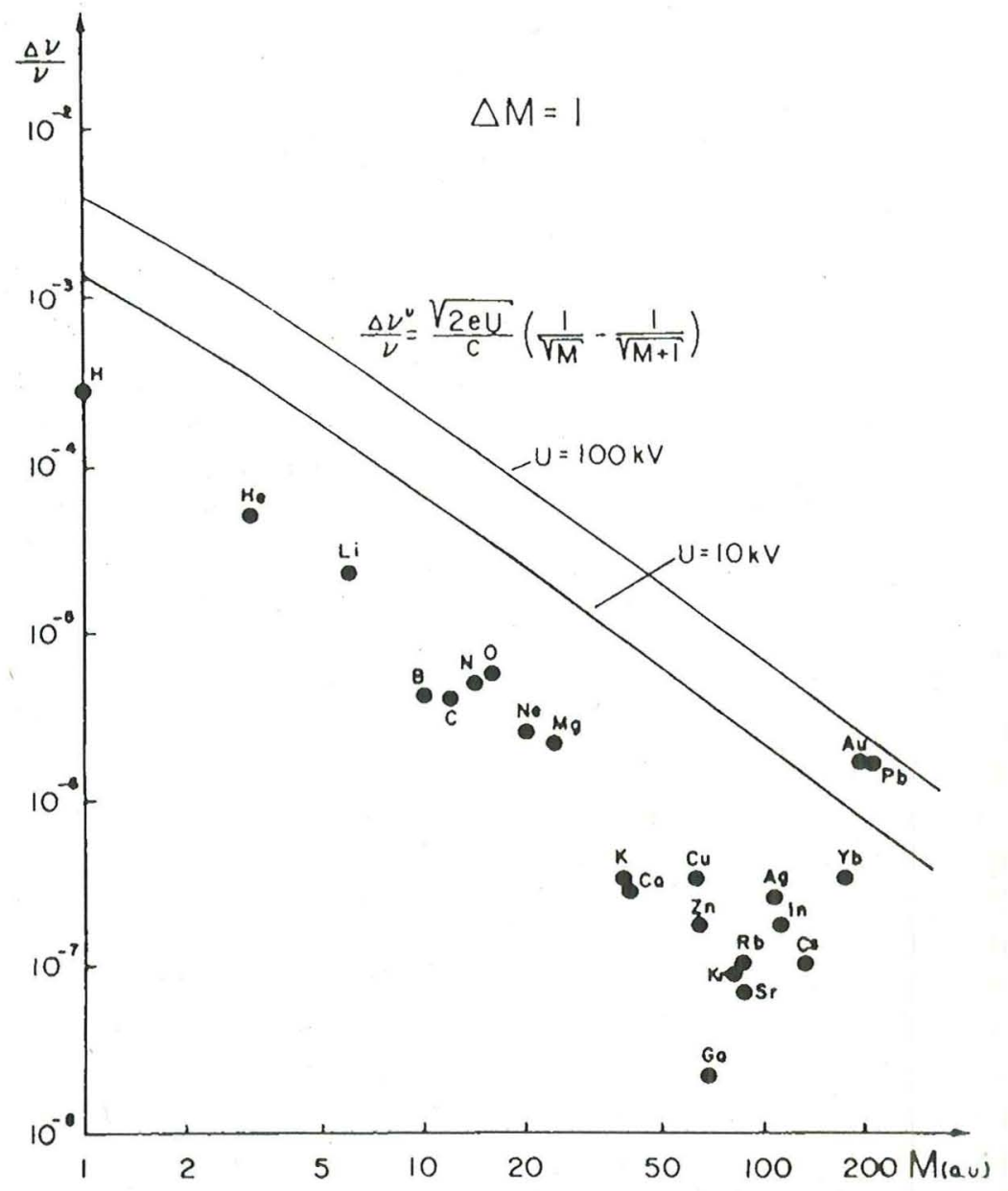

Fig. 1. Relative isotope shift of resonance lines of various elements (full dots) and kinematic isotope shift of fast atoms with an energy of $10 \mathrm{kV}$ and $100 \mathrm{kV}$ as a function of atomic mass (solid curves). 
of interest are ionized, accelcrated to an cnergy of $e U_{A}$ and neutralized in a chargeexchange cell. The atoms of the isotope under investigation are then excited isotopeselectively to a Rydberg state in a field-free region. Finally, the excited atoms are ionized by an electric field and deflected onto a detector. Since in the field-free region all the isotopic atoms have the same energy but move with different velocities, there occurs an additional isotope shift in collinear excitation. The magnitude of this shift is given by

$$
\Delta v^{\text {is }}= \pm\left(v_{0} / c\right)\left(2 e U_{A}\right)^{1 / 2}\left[1 /\left(M_{1}\right)^{1 / 2}-1 /\left(M_{2}\right)^{1 / 2}\right],
$$

where $M_{1}$ and $M_{2}$ are the masses of two isotopes, $v_{0}$ is the atomic transition frequency, and $c$ is the velocity of light. The plus and minus signs correspond to the laser light propagating with or against the atomic bcam, respectively. The solid lines in fig. 1 show this additional isotope shift (for $\Delta M=1$ ) as a function of mass for energies $e U_{A}=10$ and $e U_{A}=100 \mathrm{keV}$. The full dots indicate the natural isotope shifts for resonance transitions of various elements. It can be seen that the induced Doppler shift for atoms in the middle of the periodic table with $e U_{A}=100 \mathrm{keV}$ is 100 times their natural isotope shift. This artificial isotope shift exists in any transition of any element. Another important feature of the method is that the Doppler absorption line width of a fast atomic beam $[19,20]$ is reduced in collinear geometry by a factor of $2\left(e U_{A} / k t\right)^{1 / 2}$ and can become comparable with the natural linewidth. Hence the light of a narrow-band laser can interact with all the atoms under investigation in the fast beam. As a consequence high selectivity and sensitivity is achieved.

The method can also be applied to resonance ionization spectroscopy of atoms with high ionization potential if the atoms have high-lying metastable states to be populated in the charge exchange process.

\section{Detection of the isotope ${ }^{3} \mathrm{He}$ [16]}

The concentration of the rare isotope ${ }^{3} \mathrm{He}$ varies over a wide range relative to that of the abundant isotope ${ }^{4} \mathrm{He}$ in various objects. For example, the relative concentration of ${ }^{3} \mathrm{He}$ in the atmosphere is equal to $1.4 \cdot 10^{-6}$ and remains the same throughout the globe; it is $10^{-7}-10^{-8}$ in commercial-grade helium gas obtained from natural gas deposits and amounts only to $10^{-9}-10^{-10}$ in some minerals.

Initial experiments aiming for the detection of the rare isotope ${ }^{3} \mathrm{He}$ [21] have shown that background limits the selectivity due to collisions between the accelerated atoms of the abundant isotope ${ }^{4} \mathrm{He}$ and residual gas molecules. At a residual gas pressure in the vacuum system of $10^{-7}$ Torr, the background was equal to the signal of ${ }^{3} \mathrm{He}$ at a concentration of $10^{-6}$. To reduce the background, an additional time-offlight separation was performed of the accelerated isotopic atoms in a pulsed beam [16]. This cnabled to suppress efficiently the background signal and to detect the rare isotope ${ }^{3} \mathrm{He}$ in samples with a relative concentration as low as $10^{-9}$. 
In the presently applied technique, a beam of helium ions accelerated to an energy of $e U_{A}=3.9 \mathrm{keV}$ is produced by a duoplasmatron ion source. This beam is focused with a single electrostatic lens and then electrostatically deflected in order to separate it from the neutral component. This deflected beam passes a straight deflector used as an ion beam modulator. By applying a voltage of $U=50 \mathrm{~V}$ across the deflector plates, the ions can be completely deflected from the axis of the setup. This modulation is effected with repetition rate of copper vapor laser (see below) by means of short square pulses with a duration as short as $\tau_{\mathrm{p}}=0.5 \mu \mathrm{s}$. Downstream of the modulator, the ion beam enters a charge exchange cell containing potassium vapor. Around $40 \%$ of the ions are charge exchanged to neutral atoms. About $75 \%$ of the atoms populate the $2^{3} S_{1}$ metastable state. The ions that failed to exchange their charge and the atoms produced in highly excited Rydberg states are extracted from the beam by the electric ficld $(9 \mathrm{kV} / \mathrm{cm})$ of a filtering deflector. Neutral atoms enter a $1 \mathrm{~m}$ long field-free region where they undergo two-step laser excitation from the $2^{3} \mathrm{~S}_{1}$ metastable state to the $27^{3} \mathrm{D}_{1,2,3}$ Rydberg state via the $3^{3} \mathrm{P}_{0,1,2}$ intermediate level. Excitation is effected when a bunch of ${ }^{3} \mathrm{He}$ atoms (the bunch is $25 \mathrm{~cm}$ long at $\tau_{\mathrm{p}}=0.5 \mu \mathrm{s}$ ) is at the exit of the ficld-frec excitation region. The Rydberg atoms are ionized by a transverse electric field and deflected to a secondary electron multiplier. Such an ionization scheme of Rydberg atoms features dispersion with respect to $n$ [21]. The ${ }^{3} \mathrm{He}^{+}$ions are detected during a time interval of $\tau_{\text {det }}=0.6 \mu \mathrm{s}$ by means of a gated counting system. The background ions resulting from collisions involving ${ }^{4} \mathrm{He}$ atoms arrive at the detector delayed in time.

The Rydberg states are excited with two pulsed dye lasers. Radiation of the first step $\lambda_{1}=389 \mathrm{~nm}$ is obtained by frequency doubling in an $\mathrm{LilO}_{3}$ crystal. Radiations at $v_{1}$ and $v_{2}\left(\lambda_{2}=796 \mathrm{~nm}\right)$ are brought to overlap by means of dielectric mirrors and are carefully aligned to the accelerated atomic bcam. The dye lasers are pumped by two copper-vapor lasers, one being used as an oscillator and the other, as an amplifier.

Figure 2 presents the counting rate of ${ }^{3} \mathrm{He}$ ions as a function of the isotopic abundance in the gas. As can be scen, the function is a straight line, and the result of our measurements of ${ }^{3} \mathrm{He}$ isotopic concentrations in air and commercial-grade helium gas are in full agreement with the specified data of the samples. The counting rate of photo ions of a rare isotope at a concentration $C$ is

$$
N=I \tau_{\mathrm{P}} f \eta C,
$$

where $I$ is the continuous atomic beam current, $\tau_{\mathrm{p}}$ is the beam current pulse duration, $f$ is the laser pulse repetition rate, and $\eta$ is the efficiency of exciting the atoms and detccling the photo ions produced. For $f=8.6 \mathrm{kHz}, I=2.3 \cdot 10^{11} \mathrm{~s}^{-1}, \tau=0.5 \mu \mathrm{s}$, $C=10^{-6}$, and $\eta=0.01$, the ${ }^{3} \mathrm{He}$ photo ion counting rate is $N=10$ counts per second. The background signal corresponds to a ${ }^{3} \mathrm{He}$ concentration of $10^{-9}$ at a vacuum pressure, of $10^{-7}$ Torr in the system. The efficiency $\varepsilon$ of detecting the rare isotope atoms, determined as the ratio of the counted number $N$ of atoms and the total number of atoms in the beam $\left(N_{\text {total }}=C \cdot I\right)$, amounted to $\varepsilon=4.3 \cdot 10^{-5}$. The detection 


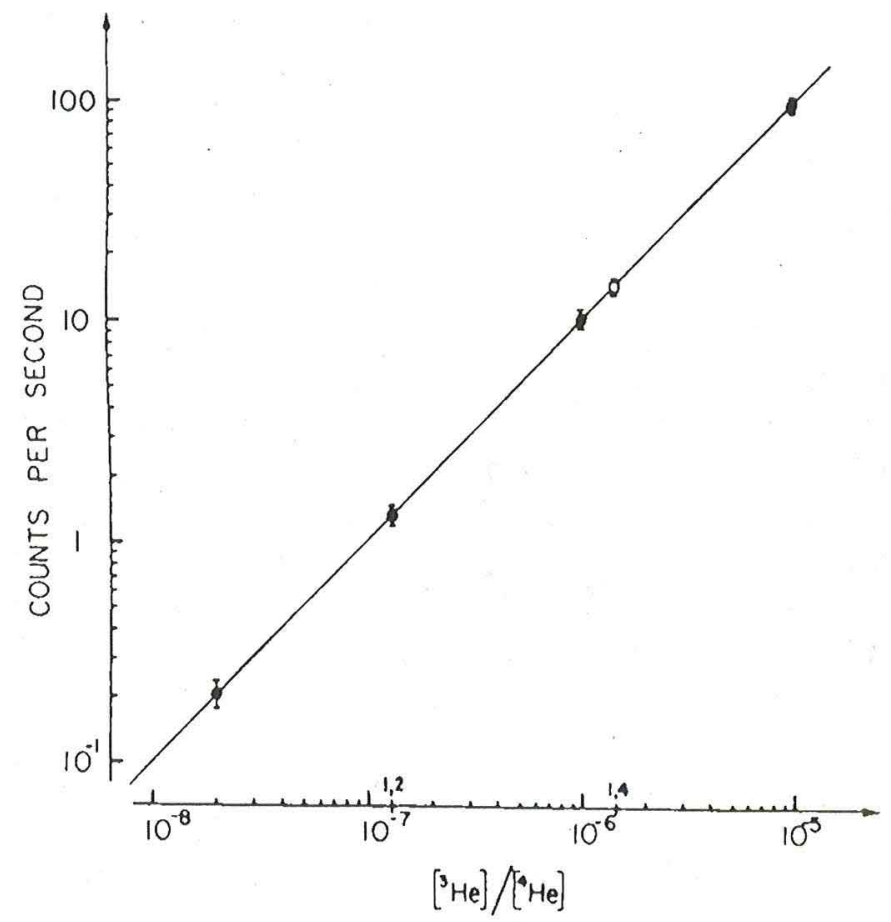

Fig. 2. Photoion signal from ${ }^{3} \mathrm{He}$ as a function of ${ }^{3} \mathrm{He}$ relative concentration. The square indicates helium gas extracted from the atmosphere.

efficiency can be improved by an order of magnitude to be $4 \cdot 10^{-4}$, by increasing the laser pulse energy to saturate the used atomic transitions. The background signal in this case will correspond to a ${ }^{3} \mathrm{He}$ concentration of $10^{-10}$.

\section{Isotope-selective ionization of krypton atoms [22]}

The detection of the rare radioactive krypton isotopes ${ }^{81} \mathrm{Kr}\left(T_{1 / 2}=2.1 \cdot 10^{5}\right.$ years, $\left.C=5 \cdot 10^{-13}\right)$ and ${ }^{85} \mathrm{Kr}\left(T_{1 / 2}=10.7\right.$ years, $\left.C=5 \cdot 10^{-11}\right)$ is of interest in environment protection as well as in studying of hydrological processes occurring on a time scale of hundreds of thousands of years.

At present, there exists one method for detecting rare krypton isotopes, based on a combination of repeated separation of the rare isotope of interest by means of a mass filter with subsequent laser resonance photo ionization detection of $\mathrm{Kr}$ atoms [23].

Experiments based on isotope-selective ionization were conducted in the setup described above [22]. Metastable krypton atoms were prepared in the $1 S_{5}\left(5 s[3 / 2]_{2}^{0}\right)$ state by quasi resonant charge exchange between krypton ions and 
potassium atoms. In a first-step at laser frequency $v_{1}$ wide-band dye laser radiation of $\Delta v_{1}=1 \mathrm{~cm}^{-1}$ counter propagating the atomic beam excited all the krypton isotopes from the $1 \mathrm{~S}_{5}$ to the $2 \mathrm{P}_{6}\left(5 \mathrm{p}[3 / 2]_{2}\right)$ level, $\lambda_{1}=7602 \AA$. The laser was pumped by the yellow line of a copper vapor laser. The second step radiation at $v_{2}$ was the green line of the copper vapor laser itself with $\lambda_{2}=5106 \AA$. This line resonated with the krypton atoms at the $2 \mathrm{P}_{6}-24 \mathrm{~d}[3 / 2]_{2}$ transition, in the case the atoms have accelerated to $2.7 \mathrm{keV}$ and the light propagating in the same direction as the atomic beam. To reduce the bandwidth $\Delta v_{2}$ to some about $0.03 \mathrm{~cm}^{-1}$, two Fabry-Pérot etalons were used with a free spectral range of $0.5 \mathrm{~cm}^{-1}$ and $0.17 \mathrm{~cm}^{-1}$. To achieve resonance in the second-step transition for different isotopes, the atomic beam was retarded by a decelerating voltage across the charge-exchange cell. The voltage of the field ionization was corrected according to the retardation voltage applied. The experimental ionization spectrum of krypton isotopes is shown in fig. 3(a). A 1-GHz change in frequency corresponds to a $31-\mathrm{eV}$ change in the atomic energy. The peaks of the even $\mathrm{Kr}$ isotopes are well resolved. The isotopes ${ }^{78} \mathrm{Kr}$, whose relative abundance is $0.35 \%$ can clearly be seen. Fig. 3(b) shows the calculated spectrum of all stable even $\mathrm{Kr}$ isotopes and of the odd isotope ${ }^{83} \mathrm{Kr}$, along with their relative concentrations. The width of the spectrum obtained is determined by the laser line width $\Delta v_{2}$ and amounts to $850 \mathrm{MHz}$.

As in the case of the isotope He, rare krypton isotopes can be detected in combination with a conventional mass separator, so that the collisional background is reduced. To implement the method, narrow-band pulsed radiation should be used in both excitation steps. This can be achieved by amplifying the radiation of a single-mode $\mathrm{cw}$ dye laser in a pulsed amplifier pumped by a copper vapor laser. Let us estimate the selectivity and sensitivity of the method using the $1 \mathrm{~S}_{5} \rightarrow 2 \mathrm{p}_{3} \rightarrow 24 \mathrm{~d}$ transitions where the hyperfine splitting constants for ${ }^{85} \mathrm{Kr}$ are known. The natural isotope shift in this transition is $50 \mathrm{MHz}$. When the atoms are accelerated to $10 \mathrm{keV}$, the isotope shift increases to $1.7 \mathrm{GHz}$. Because of the hyperfine level splitting of ${ }^{85} \mathrm{Kr}(I=9 / 2)$, its strongest line $F=13 / 2 \rightarrow F=11 / 2$ is $1.9 \mathrm{GHz}$ away from the nearest ${ }^{86} \mathrm{Kr}$ line. The laser excitation selectivity in this step is $S_{1}=2.4 \cdot 10^{5}$. The selectivity $S_{2}$ in the second step will be of the order of $10^{3}$ and the selectivity of the two-step excitation process, $S=S_{1} \cdot S_{2}$, will be at least $10^{8}$. If the conventional mass separation gives an enrichment factor of $S_{\mathrm{M}}=10^{5}$, the total selectivity will be high enough to detect both ${ }^{85} \mathrm{Kr}$ and ${ }^{81} \mathrm{Kr}$. atoms is

Let us now consider the sensitivity of the method. The counting rate for ${ }^{85} \mathrm{Kr}$

$$
N=\eta_{\mathrm{c}-\mathrm{e}} \eta_{\mathrm{cx}} \eta_{\mathrm{det}} \eta_{1 \mathrm{Ss}} I_{\mathrm{Kr}} C \tau_{\mathrm{dct}} f
$$

where $\eta_{\mathrm{c}-\mathrm{e}}=0.5$ is the charge-exchange efficiency, $\eta_{1 \mathrm{~s}_{\mathrm{s}}}=5 / 12$ the fraction of atoms in the $1 \mathrm{~S}$, state, $\eta_{\mathrm{cx}}=0.16$ the laser excitation efficiency, $\eta_{\mathrm{det}}=0.8$ the detection efficiency, $I_{\mathrm{Kr}}$ the total krypton ion current and $C$ the concentration of the isotope detected. $\tau_{\mathrm{det}}=L / v=7 \mu \mathrm{s}$ is the detection time equal to the time it takes the atoms 


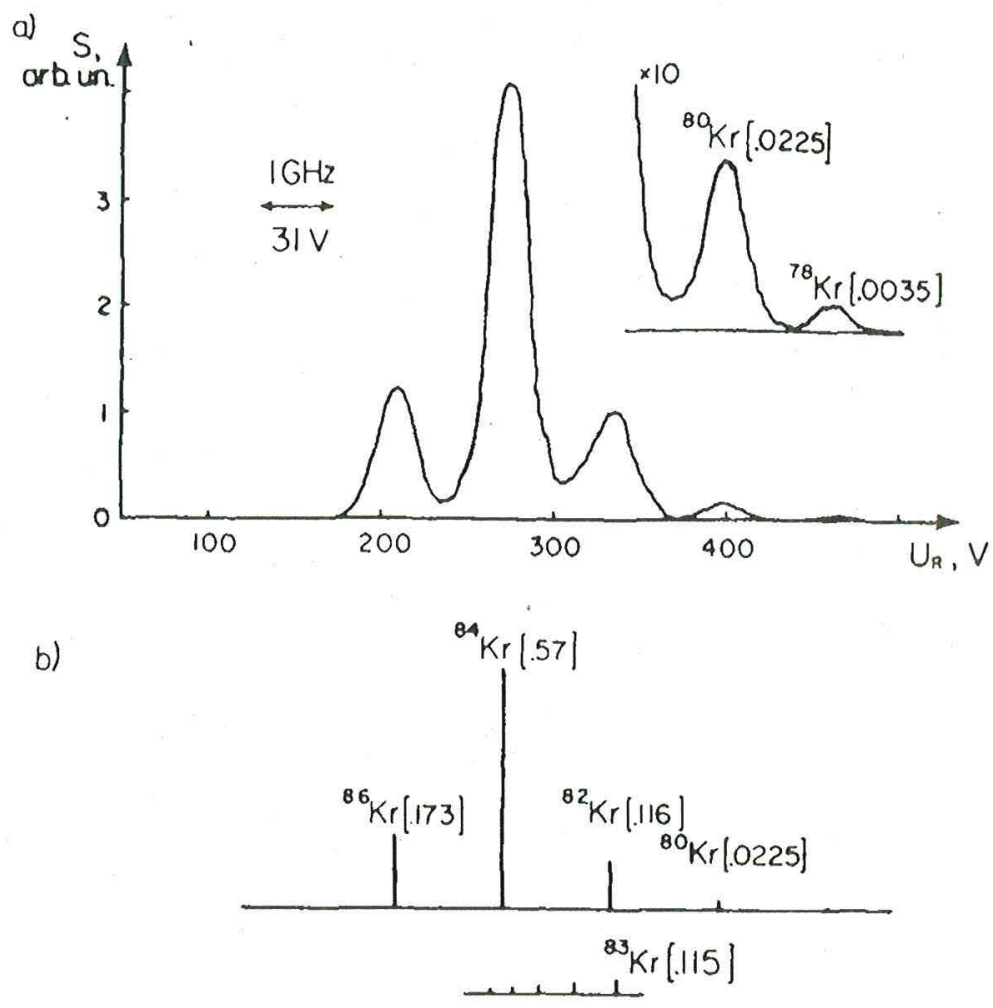

Fig. 3. (a) Photoion signal of $\mathrm{Kr}$ isotopes as a function of the charge-cxchange cell potential $\left(U_{A}=2.7 \mathrm{kV}\right)$, (b) calculated relative positions and intensities of the signals for different $\mathrm{Kr}$ isotopes.

to traverse the field-free region, and $f=10^{4} \mathrm{~Hz}$ is the laser pulse repetition rate. The counting rate for ${ }^{85} \mathrm{Kr}$ at a concentration of $5 \cdot 10^{-11}$ is 0.1 counts/s at an ion current of $I_{\mathrm{Kr}}=1.1 \cdot 10^{12}$ ions/s $(180 \mathrm{nA})$. The counting rate for the background ions produced by collisions is $N_{\mathrm{bg}}=10^{-3}$ counts/s at a vacuum of $10^{-9}$ Torr. Thus, the signal-tonoise ratio will be equal to $10^{2}$.

\section{Collinear resonance ionization spectroscopy of radioactive ytterbium atoms [24]}

The method of collinear multi-step laser ionization has been applied to measurements of isotope shifts and hyperfine structures of ytterbium isotopes produced at the ISOLDE facility at CERN. The production rate of the short living ytterbium atoms on the wing of yicld curve is less than $10^{6}$ particles/s. At the same mass the total ion current of isobars is $10^{10}$ particles/s. The applied method has allowed to measure the spectrum of rare $\mathrm{Yb}$ isotopes with an efficiency of $1 \cdot 10^{-5}$ (one detected ion per $10^{5}$ incoming atoms) and the background from collisional ionization of $1 \cdot 10^{-8}$. 
The simplified scheme of experimental setup is shown in fig. 4. The unstable $\mathrm{Yb}$ isotopes are produced in the spallation reactions ${ }^{181} \mathrm{Ta}(\mathrm{p}, 4 \mathrm{p}+\mathrm{xn}){ }^{178-x} \mathrm{Yb}$. After ionization in the ion source, acceleration to $60 \mathrm{keV}$ and mass separation the fast ytterbium ion beam is superimposed on the laser beams. The ion beam then passes through the charge-exchange cell with cesium vapor where the metastable $6 \mathrm{~s}^{2} \mathrm{p}^{3} \mathrm{P}_{0}$ state is populated. In the excitation region $(l=100 \mathrm{~cm})$ metastable atoms undergo a two-step resonance transition to one of the $6 \mathrm{~s} 21 \mathrm{p}$ Rydberg states. The atoms are finally ionized in a longitudinal electric ficld and detected.

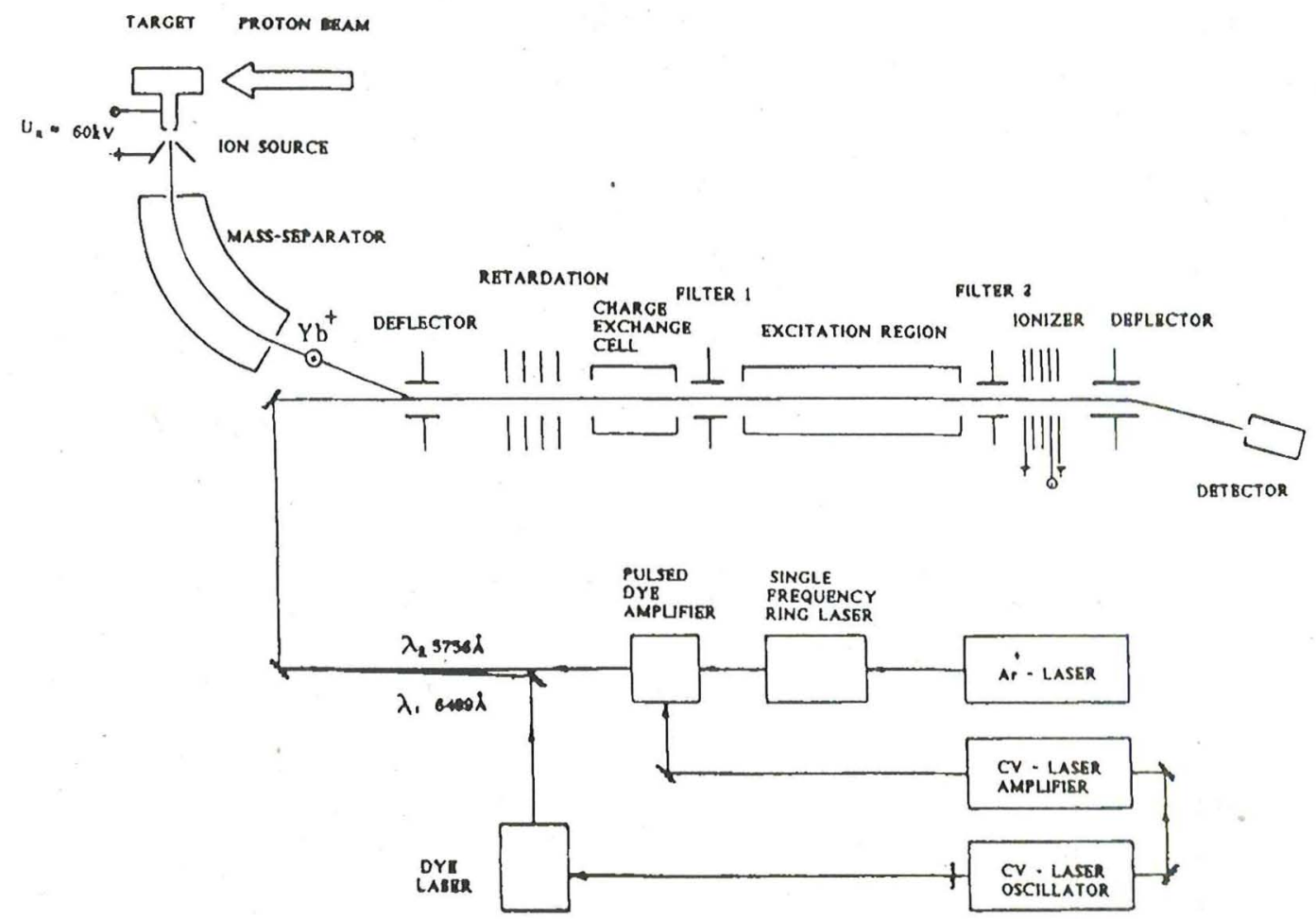

Fig. 4. Simplified scheme of expcrimental setup for laser collinear resonance ionization spectroscopy of radioactive ytterbium isotopes.

To excite the atoms from the metastable state at $\lambda_{1}=648.9 \mathrm{~nm}$, a laser with a bandwidth of $\Delta \nu_{1}=30 \mathrm{GHz}$ is used. The pulsed second-step radiation $\left(\lambda_{2}=575.6 \mathrm{~nm}\right)$ is obtained by amplifying the output of a single-frequency $\mathrm{cw}$ ring laser (Coherent CR 699/21) in a dye cell pumped by light of the copper vapor laser. The average amplified output power is $0.5 \mathrm{~W}$ and the band width $\Delta v_{2} \approx 50 \mathrm{MHz}$ corresponds to the Fourier transform of the pulse shape. The yellow CVL line is used to pump the broad-band laser, and the green line to pump the amplifier. The laser beams are transported to the apparatus over a distance of $30 \mathrm{~m}$. 
Table 1

Isotope shifts and hyperfine structure constants in the transition $6 s 7 \mathrm{~s}^{3} \mathrm{~S}_{1} \rightarrow 6 \mathrm{~s} 21 \mathrm{p}^{3} \mathrm{P}_{2}$ of the investigated ytterbium isotopes [24].

\begin{tabular}{ccccccc}
\hline$A$ & $I$ & $\begin{array}{c}I S^{168, A} \\
{[\mathrm{MHz}]}\end{array}$ & $\begin{array}{c}\delta\left\langle r^{2}\right\rangle \\
{\left[\mathrm{fm}^{2}\right]}\end{array}$ & $\begin{array}{c}A_{6 s 7 s} \\
{[\mathrm{MHz}]}\end{array}$ & $\begin{array}{c}a_{6 s} \\
{[\mathrm{MHz}]}\end{array}$ & $\begin{array}{c}\mu_{J} \\
{\left[\mu_{\mathrm{N}}\right]}\end{array}$ \\
\hline 156 & 0 & $1528(9)$ & $-1.171(24)$ & - & - & - \\
157 & $7 / 2$ & $1425(38)$ & $-1.091(6)$ & $-1260(6)$ & $-2317(24)$ & $-0.639(8)$ \\
& & $1397(31)^{1}$ & & $-1264(4)$ & $-2425(190)$ & $-0.639(8)$ \\
158 & 0 & $1238(8)$ & $-0.955(6)^{3}$ & - & - & - \\
159 & $5 / 2$ & $1101(21)^{1}$ & $-0.846(23)$ & $-1021(5)$ & $-1976(236)$ & $-0.368(8)$ \\
160 & 0 & $946(7)$ & $-0.732(4)^{3}$ & - & - & - \\
162 & 0 & $672(7)$ & $-0.516(3)^{3}$ & - & - & - \\
164 & 0 & $395(8)$ & $-0.314(3)^{3}$ & - & - & - \\
166 & 0 & $174(9)$ & $-0.140(1)^{3}$ & - & - & - \\
168 & 0 & 0 & 0 & - & - & - \\
170 & 0 & $-152(7)$ & $0.117(2)^{3}$ & - & - & - \\
171 & $1 / 2$ & $-200(13)$ & $0.157(2)^{3}$ & $6837(9)$ & $12631(84)$ & $0.49367(1)^{2}$ \\
172 & 0 & $-277(4)$ & $0.228(2)^{3}$ & - & - & - \\
173 & $5 / 2$ & $-313(20)$ & $0.266(3)^{3}$ & $-1894(8)$ & $-3505(20)$ & $-0.684(3)$ \\
174 & 0 & $-385(12)$ & $0.314(3)^{3}$ & - & - & - \\
175 & $7 / 2$ & $-402(14)$ & $0.333(13)$ & $1519(10)$ & $2803(19)$ & $0.768(8)$ \\
176 & 0 & $-482(12)$ & $0.397(4)^{3}$ & - & - & - \\
\hline & & & & & & - \\
\hline
\end{tabular}

\footnotetext{
1) Transition $6 s 7 s^{3} S_{1} \rightarrow 6 s 21 p^{3} P_{1}$

2) From [25]

3) From [26]
}

The $A$-factors of the $6 s 7 s^{3} S_{1}$ statc, $a_{6 s}$ from the analysis of the hyperfine structure (HFS) in the Rydberg states, the relative isotope shifts (IS), changes of mean square charge radii $\delta\left\langle r^{2}\right\rangle$, spins $(I)$ and magnetic moments $\left(\mu_{l}\right)$ are presented in table 1.

\section{References}

[1] V.S. Letokhov, Laser Photoionization Spectroscopy (Academic Press, Orlando, 1987) ch. 9; Comm. At. Mol. Phys. 10(1981)257.

[2] T.B. Lucatorto and J.E. Parks, in: Proc. 4th Int. Symp. on Resonance Ionization Spectroscopy and its Applications, 1988 (IOP, Bristol, 1989).

[3] V.S. Letokhov and V.I. Mishin, Opt. Commun. 29(1979)168.

[4] B.A. Bushaw, G.K. Gerke, in: Proc. 4th Int. Symp. on Resonance lonization Spectroscopy and its Applications, 1988 (IOP, Bristol, 1988) p. 277. 
[5] B.L. Fearcy, D.C. Parent, R.A. Keller and C.M. Miller, in: Proc. 4th Int. Symp. on Resonance Ionization Spectroscopy and its Applications, 1988 (IOP, Bristol, 1989) p. 285.

[6] V.I. Balykin, V.S. Lelokhov and V.I. Mishin, Appl. Phys. 22(1980)245.

[7] R.A. Keller, D.S. Bomse and D.A. Cremers, Laser Focus 17(1981)75.

[8] R.D. LaBelle, W.M. Fairbank, Jr. and R.A. Keller, Phys. Rev. A40(1989)5430.

[9] V.I. Balykin, V.S. Letokhov and V.G. Minogin, Appl. Phys. B33(1984)247.

[10] B.D. Cannon and T.J. Whitaker, Appl. Phys. B38(1985)57.

[11] G.R. Janik, B.D. Cannon, R. Ogorzalek-Loo and B.A. Bushaw, J. Opt. Soc. Amer. B6(1989)1617.

[12] A.A. Makcr, Appl. Phys. B29(1982)287; Kvantovaya Elcktronika 10(1983)1127 (in Russian).

[13] G.R. Janik, B.A. Bushaw and B.D. Cannon, Opt. Lett. 14(1989)266.

[14] Yu.A. Kudryavtsev and V.S. Letokhov, Appl. Phys. B29(1982)219.

[15] Yu.A. Kudryavtsev, V.S. Letokhov and V.V. Petrunin, Pis'ma Zh. Eksp. Teor. Fiz. 42(1985)23; [JETP Lctt. 42(1985)26].

[16] S.A. Aseyev, Yu.A. Kudryavtsev, V.S. Letokhov and V.V. Petrunin, Opt. Lett. 16(1991)514.

[17] E.W. Otten, in: Treatise on lleavy Ion Physics, Vol. 8, ed. D. Allan Bromley (Plenum Press, New York, 1988) p. 517.

[18] R. Neugart, in: Progress in Alomic Spectroscopy, Part D, ed. H.J. Beyer and H. Kleinpopen (Plenum Press, New York, 1987) p. 75.

[19] S.L. Kaufman, Opt. Commun. 17(1976)309.

[20] W.H. Wing, G.A. Ruff, W.E. Lamb and J.J. Spezewski, Phys. Rev. Lett. 36(1976)1488.

[21] Yu.A. Kudryavtsev and V.V. Petrunin, Zh. Eksp. Teor. Fiz. 94(1988)76; [Sov. Phys. JETP 67(1988)691].

[22] S.A. Ascyev, Yu.A. Kudryavtsev, V.S. Letokhov and V.V. Petrunin, J. Phys. B24(1991)2755.

[23] N. Thonard, R.D. Willis, M.C. Wright and W.A. Davis, Nucl. Instr. Meth. B29(1987)398.

[24] Ch. Schultz, E. Arnold, W. Borchers, W. Neu, R. Neugart, M. Neuroth, E.W. Otten, M. Scherf, K. Wend, P. Lievens, Yu.A. Kudryavtsev, V.S. Letokhov, V.I. Mishin and V.V. Petrunin, J. Phys. $\mathrm{B}$, to be published.

[25] L.Z. Olschewski, Z. Phys. 249(1972)205.

[26] K. Heilig, Hyp. Int. 24(1985)349. 